\title{
Loss of Cerebrovascular Autoregulation in Experimental Meningitis in Rabbits
}

\author{
Jay H. Tureen, Ronald J. Dworkin, Stephen L. Kennedy, Meena Sachdeva, and Merle A. Sande \\ Departments of Pediatrics and Medicine, University of California San Francisco, San Francisco General Hospital, \\ San Francisco, California 94110
}

\begin{abstract}
The present study was designed to determine whether cerebrovascular autoregulation is intact in experimental meningitis and to examine the relationship between fluctuations in cerebral blood flow (CBF) and increased intracranial pressure (ICP). Measurements of CBF were determined by the radionuclide microsphere technique in rabbits with experimental Streptococcus pneumoniae meningitis with simultaneous ICP monitoring via an implanted epidural catheter. CBF and ICP measurements were determined at baseline and when mean arterial blood pressure (MABP) was artificially manipulated by either pharmacologic or mechanical means. CBF was pressure passive with MABP through a range of 30-120 torr, and ICP directly correlated with CBF. These findings indicate that autoregulation of the cerebral circulation is lost during bacterial meningitis, resulting in a critical dependency of cerebral perfusion on systemic blood pressure, and that the parallel changes in ICP and in CBF suggest that fluctuations in CBF may influence intracranial hypertension in this disease. ( $J$. Clin. Invest. 1990. 85:577-581.) meningitis - cerebrovascular autoregulation • intracranial hypertension
\end{abstract}

\section{Introduction}

Autoregulation of cerebral blood flow normally is present through a wide range of mean arterial blood pressure maintaining constant cerebral perfusion in spite of transient fluctuations in blood pressure. Loss of cerebrovascular autoregulation has been described in many studies of diseases of the central nervous system, including ischemia (1), hypoxia (2), trauma (3), and metabolic disturbances (4), and has been suggested in one clinical study of patients with meningitis or encephalitis (5). In addition to the importance of intact autoregulation for provision of adequate substrate to brain tissue, increased brain blood flow may contribute to elevations in intracranial pressure by augmenting cerebral blood volume (6). The present study was designed to determine if cerebrovascular autoregulation is intact in meningitis and to examine the interrelationships between systemic blood pressure, cerebral blood flow, and increased intracranial pressure in this disease.

This work was presented in part at the Society for Pediatric Research/ American Federation for Clinical Research, 2 May 1988, Washington, DC.

Address correspondence to Dr. Jay Tureen, Department of Pediatrics, San Francisco General Hospital, 1001 Potrero Ave., San Francisco, CA 94110.

Received for publication 30 September 1988 and in revised form 6 September 1989.

J. Clin. Invest.

(c) The American Society for Clinical Investigation, Inc.

0021-9738/90/02/0577/05 $\$ 2.00$

Volume 85, February 1990, 577-581

\section{Methods}

Studies were conducted using New Zealand white rabbits $(2-2.5 \mathrm{~kg})$ Meningitis was induced by a modification of the method of Dacey and Sande (7). Rabbits were anesthetized with urethane $(2 \mathrm{~g} / \mathrm{kg})$ for all procedures, and received supplemental doses $(0.5 \mathrm{~g} / \mathrm{kg})$ to maintain light general anesthesia. A catheter (PE-100) was placed in the intracranial epidural space and arterial catheters (PE-90 or 4 Fr.; Swan-Ganz flow-directed catheter; American Hospital Supply Corporation, Irvine, CA) were inserted in the right femoral artery and left ventricle. Intracranial pressure and blood pressure were measured on a continuous basis by water-filled physiologic pressure transducers (Gould Statham P-23 XL; Gould Inc., Santa Clara, CA) and recorded on a multichannel polygraph (model 4; Grass Instrument Co., Quincy, MA). The transducers were calibrated before each experiment against a mercury-filled manometer.

Meningitis was induced by direct intracisternal injection of $\sim 1$ $\times 10^{5}$ colony-forming units of Streptococcus pneumoniae, type III, obtained from a clinical isolate. Infection was confirmed by direct plating of spinal fluid on blood agar plates incubated for $24 \mathrm{~h}$ at $37^{\circ} \mathrm{C}$ in $5 \% \mathrm{CO}_{2}$.

Arterial blood gases were obtained at baseline conditions and during blood pressure manipulations, and were measured in a clinical blood gas laboratory.

Cerebral blood flow was determined by the radionuclide-labeled microsphere technique as described by Heymann et al. (8). Microspheres, $15 \mu \mathrm{m}$ in diameter and labeled with ${ }^{153} \mathrm{Gd},{ }^{57} \mathrm{Co},{ }^{114} \mathrm{In},{ }^{51} \mathrm{Cr}$, ${ }^{113} \mathrm{Sn},{ }^{85} \mathrm{Sr},{ }^{95} \mathrm{Nb},{ }^{54} \mathrm{Mn}$, and ${ }^{65} \mathrm{Zn}$, were used. Approximately $1.5 \times 10^{5}$ microspheres were injected into the left ventricle over $30 \mathrm{~s}$ and flushed with $2 \mathrm{ml}$ of saline. The reference blood sample was withdrawn with a pump (Harvard Apparatus Co., Inc., S. Natick, MA) from the femoral catheter beginning $15 \mathrm{~s}$ before the injection, and continuing for a total of $1.5 \mathrm{~min}$ at a rate of $2.1 \mathrm{ml} / \mathrm{min}$. After all blood flow and physiologic studies were completed, rabbits were killed by injection of a lethal dose of pentobarbital $(150 \mathrm{mg} / \mathrm{kg})$ and the brain was removed by dissection from the cranial vault. The brain was dissected into right and left hemispheres, brainstem, and cerebellum, and each tissue sample was weighed in tared tubes.

Calculation of blood flow was based on the formula

$\mathrm{CBF}=\frac{\mathrm{RBF} \times C_{\mathbf{X}}}{C_{\text {arterial }}}$,

where RBF is the withdrawal rate of the reference arterial blood sample, $C_{\mathrm{X}}$ is counts per $100 \mathrm{~g}$ of brain tissues, and $C_{\text {arterial }}$ is total counts in the reference arterial blood sample. The index of autoregulatory impairment (IAI) was calculated as (\% CBF $\Delta / \%$ MABP $\Delta$ ), where CBF = cerebral blood flow (milliliters/minute per $100 \mathrm{~g}$ ) and MABP = mean arterial blood pressure. Values $\geq 1$ were considered to indicate complete loss of autoregulation, values between 0 and 1 partial loss of autoregulation, and values $\leq 0$ normal autoregulation (9).

Experimental studies. Blood flow measurements were performed 16-20 h after initial cisternal puncture in control and infected rabbits. Studies were conducted at baseline conditions, after blood pressure was increased with $l$-norepinephrine $(0.5-2 \mu \mathrm{g} / \mathrm{kg}$ per $\min$ i.v.) or by inflation of a balloon-tipped catheter in the distal aorta, and after blood

1. Abbreviations used in this paper: CSF, cerebrospinal fluid; IAI, index of autoregulatory impairment. 
pressure was lowered with sodium nitroprusside $(0.8-1.6 \mathrm{mcg} / \mathrm{kg}$ per $\mathrm{min}$ ) or hemorrhage (10-15 ml/kg) over $10 \mathrm{~min}$. Rabbits were allowed to return to baseline physiologic values for $15 \mathrm{~min}$ after pharmacologic manipulation before the next study was performed.

Data were analyzed by linear regression analysis and the paired and unpaired $t$ test. Values were considered significant when $P \leq 0.05$.

\section{Results}

Blood flow measurements were performed in 15 infected rabbits and 8 uninfected controls. In three infected and three control rabbits (group 1) two to three measurements were made after blood pressure was raised and two to three measurements made after blood pressure was lowered to evaluate cerebral hemodynamics over a broad range; in the remainder (group 2), blood pressure was raised and/or lowered and simultaneous intracranial pressure was measured. In these animals, 19 determinations were made after blood pressure was altered in the infected group and 8 in control rabbits. Blood pressure was manipulated by either pharmacologic or mechanical means and the results were not influenced by the method used. Cerebral blood flow was found to vary directly with blood pressure in all tissues studied in infected rabbits but did not change significantly in controls. When blood pressure was raised, mean change for cerebral hemispheres was 20.4 \pm 14.8 $\mathrm{ml} / \mathrm{min}$ per $100 \mathrm{~g}$ for infected rabbits $(P<0.0005)$ and $-9.8 \pm 23.3$ for control rabbits $(P=N S)$. Similar changes were observed in brainstem with infected rabbits showing an increase of $27.8 \pm 12.7(P<0.005)$ and controls a decrease of $-12.8 \pm 11.9(P=\mathrm{NS})$ and in cerebellum $25.2 \pm 19(P<0.005)$ vs. $-11.0 \pm 25.9(P=\mathrm{NS})$. When blood pressure was reduced, mean change in cerebral hemispheric blood flow was $-22.9 \pm 13.7$ in infected $(P<0.0005)$ and $-10.2 \pm 32.1$ in control $(P=\mathrm{NS})$; change in brainstem blood flow was $-17.9 \pm 15.2$ $(P<0.005)$ vs. $-2.5 \pm 32.1(P=\mathrm{NS})$; and change in cerebellar blood flow was $-23.3 \pm 25.2(P<0.05)$ vs. $-13.7 \pm 34.7$ ( $P$ $=$ NS). These data are summarized in Table $I$.

Arterial blood gas values were similar between the control and infected animals. Baseline values were $\mathrm{pH} 7.36 \pm 0.06$, $\mathrm{PACO}_{2} 32 \pm 9$ torr, and $\mathrm{PA}_{\mathrm{O}_{2}} 83 \pm 17$ torr for controls and $7.37 \pm 0.05,35 \pm 7$, and $84 \pm 21$ for infected animals. When blood pressure was elevated the values were $7.37 \pm 0.05,30 \pm 6$, and $99 \pm 32$ for controls and $7.42 \pm 0.10,30 \pm 10$, and $94 \pm 26$ for infected animals. When blood pressure was lowered the values were $7.32 \pm 0.11,30 \pm 4$, and $85 \pm 32$ for controls and $7.39 \pm 0.04$, $31 \pm 7$, and $71 \pm 33$ for infected animals. While these demonstrate mild hyperventilation with blood pressure manipulation, there were no significant differences between infected and control rabbits.

Autoregulation was qualitatively assessed in group 1 animals by constructing individual autoregulatory curves for each animal by serial blood pressure manipulation with multiple determinations. All infected animals and one control rabbit demonstrated loss of autoregulation at reduced blood pressure above the autoregulatory threshold. When blood pressure was further reduced below the lower limits of autoregulation, all infected and one control demonstrated reduced perfusion. With blood pressure elevation there was a progressive increase in blood flow in infected animals which was not observed in the controls; however, it was not possible to document the upper limit of autoregulation in this study due to drug toxicity or failure of mechanical means to sufficiently elevate blood pressure to that level.

Autoregulation was quantitatively assessed in group 2 by calculation of the IAI for each paired determination. Results for each tissue from control and infected rabbits are shown in Table II. When these rabbits were examined individually, 1 of 8 manipulations in control rabbits and 17 of 19 manipulations in infected rabbits showed an IAI of $\geq 1$ in cerebral hemispheres. Similar results were found in brainstem (impaired autoregulation in 1 of 8 control and 15 of 19 infected rabbits) and cerebellum (impaired autoregulation in 2 of 8 control and 14 of 19 infected rabbits). The two determinations in infected rabbits where hemispheric autoregulation was not completely impaired were 0.83 and 0.97 , demonstrating partial loss of autoregulation, whereas this was not observed in the uninfected control animals. Composite data (groups 1 and 2) depicting the relationship between blood pressure and cerebral hemispheric blood flow for both blood pressure elevation and reduction are presented in Figs. 1 and 2. With increased blood pressure, measurements in 15 of 15 infected and 3 of 8 control rabbits demonstrated increased blood flow. With reduced blood pressure, measurements in 11 of 11 infected and only 1 of 6 control rabbits showed reduction in blood flow at hypotensive levels that were above the autoregulatory threshold.

The influence of fluctuating arterial pressure on intracranial pressure was determined concomitantly in group 2 rab-

Table I. Cerebral Blood Flow (CBF) and Intracranial Pressure (ICP) in Experimental Meningitis

\begin{tabular}{|c|c|c|c|c|}
\hline & $\mathrm{CBF}_{\text {ceretorum }}$ & $\mathrm{CBF}_{\mathrm{BS}}$ & $\mathrm{CBF}_{\text {carenoflum }}$ & ICP \\
\hline & & $\mathrm{ml} / \mathrm{min}$ per $100 \mathrm{~g}$ tissue & & $\mathrm{mmHg}$ \\
\hline \multicolumn{5}{|l|}{ Infected $(n)$} \\
\hline Baseline (12) & $63.5 \pm 20$ & $79.1 \pm 23.4$ & $85.7 \pm 40.8$ & $16.1 \pm 15.5$ \\
\hline $\mathrm{BP}$ raised (11) & $83.9 \pm 27.6^{*}$ & $106.9 \pm 38.5^{\ddagger}$ & $110.9 \pm 63^{\ddagger}$ & $19.3 \pm 13.8^{8}$ \\
\hline BP lowered (8) & $40.4 \pm 20.5^{*}$ & $61.2 \pm 26.7^{\ddagger}$ & $62.4 \pm 35.1^{11}$ & $15.6 \pm 12.5^{*}$ \\
\hline \multicolumn{5}{|l|}{ Control $(n)$} \\
\hline Baseline (5) & $67.2 \pm 19.6$ & $81.8 \pm 10.8$ & $73.4 \pm 32.4$ & $3 \pm 2.2$ \\
\hline BP raised (5) & $57.4 \pm 14.6$ & $69 \pm 18.9$ & $62.4 \pm 20.2$ & $2.4 \pm 3.3$ \\
\hline BP lowered (3) & $57 \pm 17.1$ & $79.3 \pm 42.6$ & $59.7 \pm 5.1$ & $3.3 \pm 4.2$ \\
\hline
\end{tabular}

Values expressed as mean $\pm 1 \mathrm{SD}$. $\mathrm{CBF}_{\text {cerebrum }}, \mathrm{CBF}_{\mathrm{BS}}$, and $\mathrm{CBF}_{\text {cerebellum }}$ are cerebral blood flow in cerebral hemisphere, brainstem, and cerebellum, respectively. ${ }^{*} P<0.0005$ compared with baseline; ${ }^{\ddagger} P<0.005$ compared with baseline; $\$ P<0.05$ compared with baseline; $\quad P<$ 0.01 compared with baseline (paired $t$ test). 
Table II. IAI in Brain Tissues in Experimental Meningitis

\begin{tabular}{|c|c|c|c|}
\hline$n$ & IAI $I_{\text {oencerum }}$ & $\mathrm{IAI}_{\mathrm{BS}}$ & IA $\mathrm{I}_{\text {centedellum }}$ \\
\hline Infected (19) & $2.12 \pm 1.2^{*}$ & $1.95 \pm 1.33^{\ddagger}$ & $1.48 \pm 1^{8}$ \\
\hline Control (8) & $-0.02 \pm 0.28$ & $-0.25 \pm 0.86$ & $-1.07 \pm 2.13$ \\
\hline
\end{tabular}

Values expressed as mean \pm 1 SD.

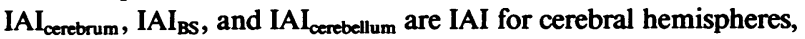
brainstem, and cerebellum, respectively.

${ }^{*} P<0.001$ compared with control; ${ }^{\ddagger} P<0.005$ compared with control; ${ }^{\S} P<0.01$ compared with control (unpaired $t$ test).

bits. Elevation of blood pressure was associated with a mean increase in intracranial pressure of $3.2 \pm 5.7 \mathrm{mmHg}$ in infected rabbits $(P<0.05)$ but had no effect in uninfected controls $(-0.6 \pm 1.3 ; P=\mathrm{NS})$. When blood pressure was reduced, mean intracranial pressure fell by $-0.5 \pm 8.6$ in infected $(P<0.05)$ and rose $0.3 \pm 1.5$ in controls $(P=N S)$. In 15 of 19 manipulations in infected rabbits intracranial pressure changes occurred in concert with the change in blood pressure, whereas parallel changes occurred in only 2 of 8 manipulations in controls. Composite data showing the relationship between cerebral blood flow and intracranial pressure for both blood pressure elevation and reduction are depicted in Figs. 3 and 4. Figs. 5 and 6 show the effect of change in cerebral blood flow on intracranial pressure for paired determinations in individual rabbits.

\section{Discussion}

Bacterial meningitis is known to be associated with a number of physiologic disturbances of the central nervous system, including reduction in cerebral perfusion $(5,10)$, increased intracranial pressure (11), metabolic alterations (12), and brain edema $(11,13)$. Previous studies by Smith (10) have demonstrated decreased perfusion in monkeys infected with a pathogenic strain of Hemophilus influenzae compared with monkeys infected with a nonpathogenic strain. Studies by Paulson et al. (5) in human subjects with meningitis due to $S$. pneumoniae or Neisseria meningitidis, or encephalitis of presumed viral etiology, demonstrated reduction with $S$. pneumoniae or encephalitis. Regional cerebral blood flow was determined by ${ }^{133} \mathrm{Xe}$ washout, and when blood pressure was elevated by angiotensin infusion, partial loss of autoregulation was demonstrated with $S$. pneumoniae and with encephalitis (IAI = 0.57), but not with $N$. meningitidis.

The present study demonstrates loss of cerebrovascular autoregulation in rabbits with meningitis in response to induced

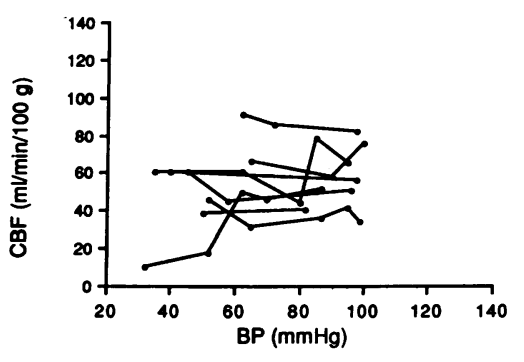

Figure 1. Cerebral blood flow $(C B F)$ as a function of mean arterial blood pressure in uninfected rabbits. No significant relationship is noted between the two parameters. $y$ $=54.01+0.10 x, r$ $=0.12, P>0.05$.

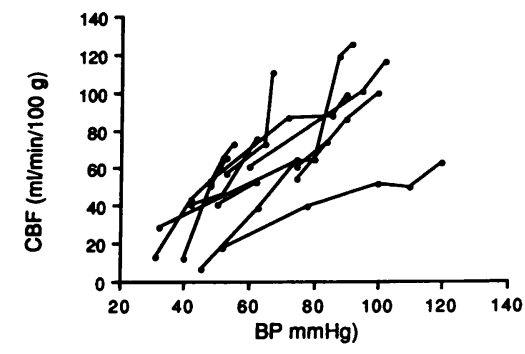

Figure 2. Cerebral blood flow $(C B F)$ as a function of mean arterial blood pressure in infected rabbits. CBF shows a highly significant association with mean arterial blood pressure throughout the range of pressures, indicating perfusion that is pressure passive. $y=33$

$+0.56 x ; r=0.81, P$ $<0.0005$.

changes in blood pressure. This phenomenon was not observed in control rabbits, and was consistently observed whether pharmacologic or mechanical means were used to change blood pressure, indicating that the circulatory changes were not an artifact of the preparation or a result of the anesthetic agent or other drugs used to manipulate blood pressure. Although we noted variability in baseline blood flow in individual rabbits, what is notable is the consistent response observed in each infected animal to changes in blood pressure.

These alterations in cerebral blood flow do not seem to be due to changes in arterial $\mathrm{CO}_{2}$ concentration, which also influences cerebrovascular tone. Infected, hypertensive animals showed an increase in blood flow in spite of a reduction in $\mathrm{PACO}_{2}$, and decreased perfusion with hypotension at identical $\mathrm{PA}_{\mathrm{CO}_{2}}$ levels. Infected animals, excluding those measurements obtained below the lower limit of autoregulation, failed to maintain constant perfusion when blood pressure was manipulated from mean arterial blood pressure of 40-120 torr, a range where autoregulation has been previously noted to be present in this species (14).

Autoregulation is the mechanism whereby organ blood flow is maintained at constant levels by changes in vascular resistance in response to changes in perfusion pressure. Cerebrovascular autoregulation occurs through dilatation or constriction of cerebral resistance vessels in response to alterations in cerebral perfusion pressure, either due to changes in mean arterial blood pressure or intracranial pressure. As autoregulation has been found to be impaired in a number of insults to the central nervous system, it is not surprising that it is disordered in meningitis; however, the implications are of critical importance in management of patients with meningitis. Impairment of the normal mechanism for matching perfusion

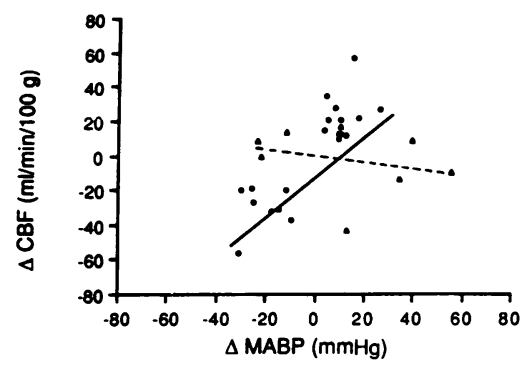

Figure 3. Change in cerebral blood flow $(\triangle C B F)$ as a function of change in blood pressure $(\triangle M A B P)$ in control $(---\Delta-\cdot)$ and infected $(-\bullet-)$ rabbits. Changes in MABP result in corresponding changes in CBF in infected rabbits but do not cause similar changes in controls. 


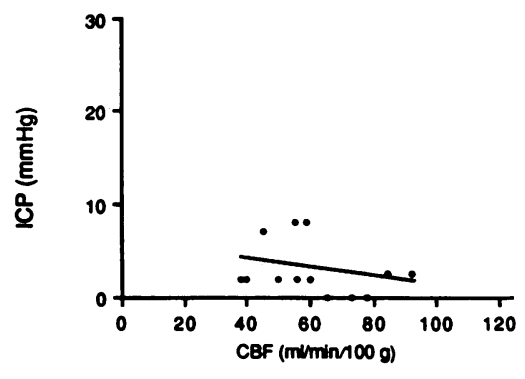

Figure 4. Intracranial pressure. $(I C P)$ as a function of cerebral blood flow $(C B F)$ in uninfected controls. No significant relationship exists between the two parameters. $y=6.59$ $-0.06 x, r=-0.35$, $P=$ NS.

with metabolic demand leaves the brain at risk for significant hypo- or hyperperfusion, both of which may have adverse consequences. While the effects of hypoperfusion are obvious, hyperperfusion may also have deleterious consequences. Lou (15) proposed that transient increases in cerebral blood flow in response to increased cerebral perfusion pressure might be in part responsible for subependymal hemorrhage in neonates, resulting from increased transmural pressure that is not buffered by the expected increase in cerebrovascular resistance. In addition, our observation that intracranial pressure increased with increased blood flow raises the possibility that cerebral blood volume may contribute significantly to intracranial hypertension in patients with meningitis.

Intracranial pressure elevations in meningitis are not completely understood and may be due to increases in any intracranial component: cerebrospinal fluid (CSF), brain tissue water, or cerebral blood volume. Scheld et al. (16) demonstrated increased resistance to CSF outflow in meningitis, probably due to arachnoid membrane dysfunction (17) with diminished resorption of CSF through the arachnoid granulations. However, intracranial pressure elevations from increased CSF volume should occur gradually and would not explain the acute increases we observed in association with changes in blood pressure. Increased brain water content as a consequence of brain edema may also contribute; however, the studies of Täuber et al. (11) and others have demonstrated a dissociation between increased brain water content and increased intracranial pressure. In the studies by Täuber et al., dexamethasone and methylprednisolone reduced brain edema; however, only dexamethasone simultaneously reduced intracranial pressure. Similar results were obtained by Tureen et al. (18), where indomethacin reduced brain edema but not intracranial pressure. These results suggest that pathophysiologic changes other than brain edema may have a role in intracranial hypertension in meningitis.

The present study demonstrates that transient changes in cerebral perfusion contribute to changes in intracranial pressure in meningitis. Infected animals showed intracranial pressure that changed in parallel with cerebral blood flow and this

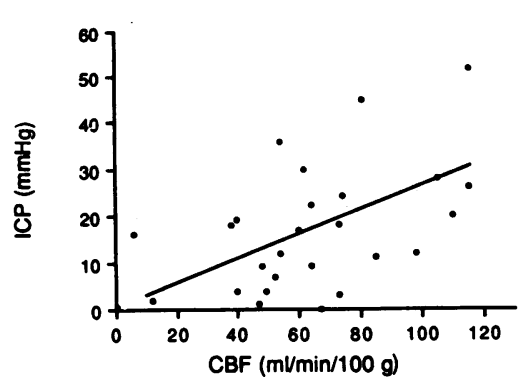

Figure 5. Intracranial pressure $(I C P)$ as a function of cerebral blood flow $(C B F)$ in infected rabbits. ICP shows a significant association with $\mathrm{CBF}$ throughout the range of measured values. $y$ $=0.52+0.26 x ; r=$ $0.50, P<0.01$.

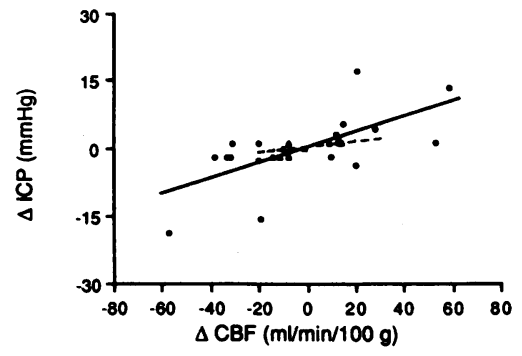

Figure 6. Change in intracranial pressure $(\triangle I C P)$ as a function of change in cerebral blood flow $(\triangle C B F)$ in control (---A---) and infected $(-\bullet-)$ rabbits. Changes in CBF induced by manipulation of blood pressure are reflected in parallel changes in ICP in most instances in infected rabbits, but are not seen in controls.

phenomenon was not observed in controls. It is likely that loss of brain compliance, which may occur as a result of brain edema, is responsible for the increased sensitivity to small changes in intracranial blood volume. Support for this is drawn from the studies of Leech and Miller (19), which demonstrated that when brain compliance was reduced by progressive inflation of an intracranial balloon, small changes in intracranial blood volume resulted in marked elevations in intracranial pressure. A similar observation was reported by Muizelaar et al. (6), who described 42 head-injured patients in whom autoregulation was studied. 14 patients had defective autoregulation as demonstrated by increased or decreased cerebral blood flow in response to pharmacologically induced increases or decreases in blood pressure. Patients in whom autoregulation was defective showed a concomitant increase or decrease in intracranial pressure when blood flow was augmented or reduced, whereas this was not observed in patients where autoregulation was preserved.

The clinical implications of these observations could be very significant in the management of patients with bacterial meningitis. These patients have frequent fluctuations in physiologic parameters due to anxiety, pain, dysfunction of the central nervous system, or as a consequence of intravascular volume abnormalities due either to inappropriate secretion of antidiuretic hormone or inappropriate fluid administration. With loss of cerebrovascular autoregulation, fluctuation in mean arterial blood pressure is likely to have adverse consequences for patients with meningitis, with risk of brain injury from either transient hypotension or hypertension. Hypotensive episodes that lead to reduced cerebral perfusion pressure may result in periods of inadequate blood flow, which would contribute to neuronal injury. The study of Goitein and Tamir (20) supports the hypothesis that cerebral perfusion pressure is a critical determinant of both short- and long-term outcome. They found that low cerebral perfusion pressure was strongly correlated with death or neurologic injury. In that study, isolated intracranial pressure elevation when accompanied by a corresponding increase in mean arterial blood pressure which maintained cerebral perfusion pressure was not predictive of poor outcome. On the other hand, a hypertensive episode may also be injurious if it causes a spike in intracranial pressure. With impaired autoregulation, blood pressure elevation leads to increased cerebral blood flow with resultant elevation of intracranial pressure, and intracranial hypertension in meningitis may result in cerebral herniation (21).

These observations highlight the interrelationship between blood pressure, cerebral perfusion, and intracranial hypertension, and strongly support careful fluid management, intracra- 
nial pressure, and blood pressure monitoring in patients with bacterial meningitis. It appears that there may be a relatively narrow range of cerebral perfusion pressure required for adequate cerebral blood flow, but which does not aggravate intracranial hypertension. Patients with bacterial meningitis should be managed with measures that will maintain cerebral perfusion pressure at a level of $50-70 \mathrm{mmHg}$, as suggested for other forms of central nervous system injury (22).

\section{Acknowledgments}

This work was supported by grants from the National Institutes of Health (NS-27310) and Hoffman-LaRoche, Inc.

\section{References}

1. Heistad, D. D., M. L. Marcus, D. J. Piegors, and M. L. Armstrong. 1980. Regulation of cerebral blood flow in atherosclerotic monkeys. Am. J. Physiol. 239:H539-H544.

2. Kogure, K., P. Scheinberg, M. Fujishima, R. Busto, and O. M. Reinmath. 1970. Effects of hypoxia on cerebral autoregulation. Am. J. Physiol. 219:1393-1396.

3. Enevoldsen, E. M., and F. T. Jensen. 1978. Autoregulation and $\mathrm{CO}_{2}$ responses of cerebral blood flow in patients with acute severe head injury. J. Neurosurg. 48:689-703.

4. Ong, B. Y., R. Greengrass, D. Bose, G. Gregory, and R. J. Palahniuk. 1986. Acidemia impairs autoregulation of cerebral blood flow in newborn lambs. Can. Anaesth. Soc. J. 33:5-9.

5. Paulson, O. B., P. Brodersen, E. L. Hansen, and H. S. Kristensen. 1974. Regional cerebral blood flow, cerebral metabolic rate of oxygen, and cerebrospinal fluid acid-base variables in patients with acute meningitis and with acute encephalitis. Acta Med. Scand. 196:191-198.

6. Muizelaar, J. P., H. A. Lutz, and D. P. Becker. 1984. Effect of mannitol on ICP and CBF and correlation with pressure autoregulation in severely head-injured patients. J. Neurosurg. 61:700-706.

7. Dacey, R. G., and M. A. Sande. 1974. Effect of probenecid on cerebrospinal fluid concentrations of penicillin and cephalosporin derivatives. Antimicrob. Agents Chemother. 6:437-441.

8. Heymann, M. A., B. D. Payne, J. I. E. Hoffman, and A. M. Rudolph. 1977. Blood flow measurements with radionuclide-labeled particles. Prog. Cardiovasc. Dis. 20:55-70.

9. Tweed, A., J. Cote, G. Gregory, and J. Wade. 1986. Impairment of cerebral blood flow autoregulation in the newborn lamb by hypoxia. Pediatr. Res. 20:516-519.
10. Smith, A. L. 1983. Pathogenesis of Haemophilus influenzae type b meningitis. In Experimental Bacterial and Parasitic Infections. G. Deusch and T. Wadstrom, editors. Elsevier Science Publishing, New York. 295-301.

11. Täuber, M. G., H. Khayam-Bashi, and M. A. Sande. 1985. Effects of ampicillin and corticosteroids on brain water content, cerebrospinal fluid pressure, and cerebrospinal fluid lactate levels in experimental pneumococcal meningitis. J. Infect. Dis. 151:528-534.

12. Brook, I., K. S. Bricknell, G. D. Overturf, and S. M. Feingold. 1978. Measurement of lactic acid in cerebrospinal fluid of patients with infections of the central nervous system. J. Infect. Dis. 137:384390.

13. Dodge, P. R., and M. N. Swartz. 1965. Bacterial meningitis: a review of selected aspects. $N$. Engl. J. Med. 272:954-960.

14. Tuor, U. I., and J. K. Farrar. 1984. Pial vessel caliber and cerebral blood flow during hemorrhage and hypercapnia in the rabbit. Am. J. Physiol. 247:H40-H51.

15. Lou, H. C. 1980. Perinatal hypoxic-ischemic brain damage and intraventricular hemorrhage. Arch. Neurol. 37:585-587.

16. Scheld, W. M., R. G. Dacey, H. R. Winn, J. E. Walsh, J. A. Jane, and M. A. Sande. 1980. Cerebrospinal fluid outflow resistance in rabbits with experimental meningitis. Alterations with penicillin and methylprednisolone. J. Clin. Invest. 66:243-253.

17. Quagliavello, V., W. Long, and W. M. Scheld. 1986. Morphologic alterations of the blood-brain barrier with experimental meningitis in the rat. Temporal sequence and role of encapsulation. J. Clin. Invest. 77:1084-1095.

18. Tureen, J. H., F. B. Stella, R. I. Clyman, F. Mauray, and M. A. Sande. 1987. Effect of indomethacin on brain water content, cerebrospinal fluid white blood cell response, and prostaglandin $E_{2}$ levels in cerebrospinal fluid in experimental pneumococcal meningitis in rabbits. Pediatr. Infect. Dis. J. 6(Suppl):1151-1153.

19. Leech, P., and J. D. Miller. 1974. Intracranial volume-pressure relationships during experimental brain compression in primates. 2 . Effect of induced changes in systemic arterial pressure and cerebral blood flow. J. Neurol. Neurosurg. Psychiatry. 37:1099-1104.

20. Goitein, K. J., and I. Tamir. 1983. Cerebral perfusion pressure in central nervous system infections of infancy and childhood. $J$. Pediatr. 103:40-43.

21. Horwitz, S. J., B. Boxerbaum, and J. O'Bell. 1980. Cerebral herniation in bacterial meningitis in childhood. Ann. Neurol. 7:524528.

22. Dacey, R. G. 1987. Monitoring and treating increased intracranial pressure. Pediatr. Infect. Dis. J. 6:1161-1163. 